\title{
Performance Analysis of Different Micro Ring Resonators based on Optical Delay Lines
}

\author{
P.Narmadhadevi \\ Assistant Professor \\ Sri Ramakrishna Institute of \\ Technology \\ Coimbatore, Tamilnadu, India
}

\author{
D.Shanmuga Sundar \\ Research Scholar \\ Alagappa Chettiar College of \\ Engineering and Technology \\ Karaikudi, Tamilnadu, India
}

\author{
L.Malathi \\ Assistant Professor \\ Sri Ramakrishna Institute of \\ Technology \\ Coimbatore, Tamilnadu, India
}

\begin{abstract}
This paper, deals with design and simulation of optical delay line consisting of an array of micro-ring resonator. The work comprises the comparison of three types of delay lines namely SCISSOR, CROW and Ring-loaded MZT interferometer based delay line. Loaded MZT interferometer configuration consists of array of micro-ring resonators connected to the upper arm. In SCISSOR configuration rings are cascaded serially and the light input at the bus waveguide is evanescently coupled to the ring waveguide. In CROW light input at the bus waveguide is coupled to the ring and is also coupled between the rings. In this paper comparison of the temporal parameters such as overall delay, group delay and also the spectral characteristics such as Free Spectral Range, loss and rejection line width are made. The delay achieved by this design is on the order of picoseconds which can be used in the OTDM networks for demultiplexing the channels and in Antenna Beam Forming networks.
\end{abstract}

\section{Keywords}

Micro ring resonator, Optical delay line.

\section{INTRODUCTION}

Slow light devices have important applications in the areas of buffering, optical signal processing and phased array antenna. Dynamic optical buffers which produce variable optical delay lines are the key elements in optical signal processing systems and future optical networks. These delay line devices are mainly used in optical packet switches. They are also used as optical filters, add drop multiplexers and as beam formers in phased array antenna. They are called as "true time delay" devices in microwave circuitry. In optical packet switching applications, buffers are used for synchronization of incoming packets and for collision avoidance on outgoing light paths. In earlier days fiber delay lines are used for collision avoidance by passing the packets into the fiber thereby delaying the packet for the time equal to the length of the fiber. But it is bulky and does not scale well to full-size networks, and hard to produce on-chip memory.

Different schemes have been proposed the implementation of optical delay lines as following
i) Fiber delay lines
ii) Electro-Magnetically Induced Transparency
iii) Coupling Micro-ring resonators

Among the aforementioned techniques this paper deals with array of micro ring resonator structure coupled to a bus waveguide in three different ways.

\section{MICRO-RING RESONATOR AS MEMORY}

High Optical ring resonators are used to selectively filter, switch or modulate the light beams. They have an optical waveguide in a closed loop coupled to a tangential waveguide. A light having wavelength of resonance is coupled to the ring and the other wavelength are transmitted unaffected.

The wavelength which is coupled to the ring will be circulated/delayed for the required time and is then detuned to be transmitted [1]. By this way it acts as a memory device. The device is called as All Pass Filter (APF). The resonator introduces a phase delay as the light passed through the ring. If the incoming signal is periodic, i.e. train of pulse, then the spectrum of the pulse consists of discrete components spaced by the repetition rate of the pulse. After passing through the APF the delayed spectrum is periodic. The period of the spectrum is called as Free Spectral Range of APF. When the period of the pulse is made equal to FSR all spectral components experience constant group delay.

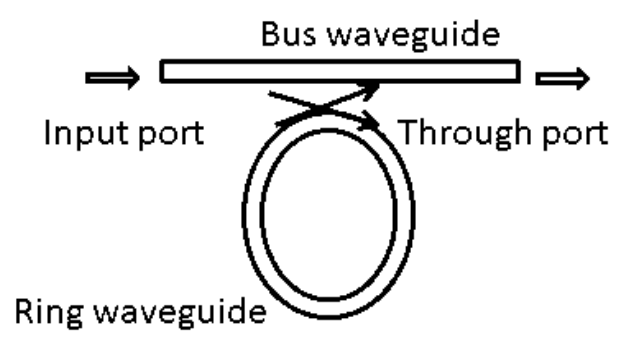

Fig 1: Waveguide coupled to lossy ring working as All

Pass Filter. The APF induces a phase shift $\varphi(\omega)$ in an input field Ein $(\omega)$ and introduces a group delay $\tau \mathrm{g}$ on an optical pulse Ein (t) of duration T0 given at the input port. The delayed pulse is noted in through port

In time domain analysis, if the incoming signal is a train of pulse, when it is coupled to the ring and has completed its round trip in APF configuration, it interfere with the next pulse in the train constructively[2].

\subsection{Different Types of Delay Lines}

There are three types of delay lines in literature.

i) SCISSOR (Side-Coupled Integrated Space Sequence of Resonators)

ii) CROW (Coupled Resonator Optical Waveguides)

iii) Loaded MZT (Loaded Machzhender Interferometer)

Micro ring resonators are highly dispersive elements. A single micro ring resonator usually does not provide adequate group delay or bandwidth for most practical 
applications. One way to increase this bandwidth-delay product is by cascading multiple stages of micro rings to form linear sequences such as CROWs or SCISSOR. By using cascaded structure flat group delay can be achieved. These structures acts both as delay lines and band pass filter $[3,6]$.

Among the factors affecting the performance of a SOI cascaded micro ring slow light device, the gaps between the bus and the ring waveguides and the distances between the adjacent rings are much more important [4,7] because they determine the spectrum shape and therefore the delay time and wavelength of the device.

\section{DESIGN AND OPTIMIZATION OF DELAY LINES}

This paper compares the various results of SCISSOR,CROW and loaded MZT structure. Figure 2 shows the loaded MZT and figure 3 shows SCISSOR. Both the structure consists of 6 micro rings. The device is fabricated on SOI wafer with $0.3 \mu \mathrm{m}$ thick silicon and $3 \mu \mathrm{m}$ silica as substrate and $1.5 \mu \mathrm{m}$ air as cladding layer [5] (Figure. 1).

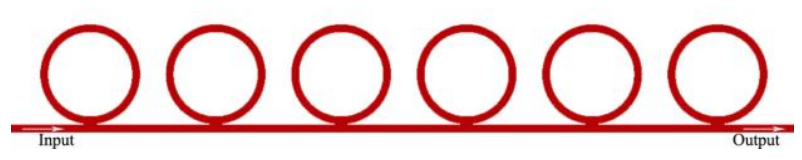

(a)

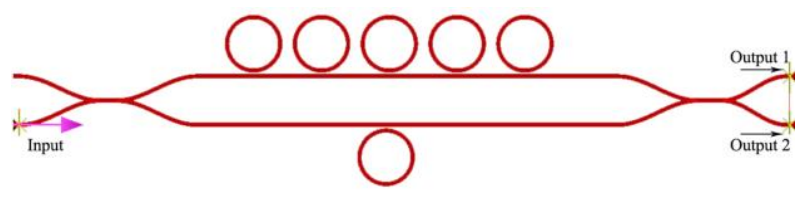

(b)

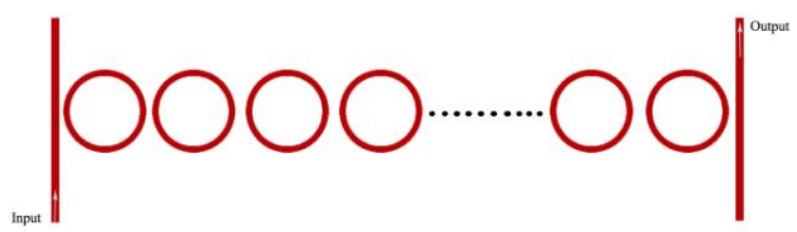

(c)

Fig 2: Schematic of the Optical delay lines a) SCISSOR, b) Loaded MZT, c) CROW with ring radius $1.5 \mu \mathrm{m}$ and the width of both bus and ring waveguide of $0.3 \mu \mathrm{m}$

The radii of micro rings are $1.5 \mu \mathrm{m}$ and the gap between the rings is $500 \mathrm{~nm}$. The gap between the ring and the bus waveguide is $100 \mathrm{~nm}$. Here the bus and ring waveguide of width of $300 \mathrm{~nm}$ is used. For this dimension a delay of $0.8 \mathrm{ps}$ is achieved. If the width of the ring is more than that of the bus waveguide then the delay will be high.

\section{RESULT AND DISCUSSIONS}

The experiment is done for rings of radius equal to the integer multiple of wavelengths of the input signal.

\section{Output For Rings Of Increasing Radius In Time Domain}

Ring radius $=1.5 \mu \mathrm{m}$
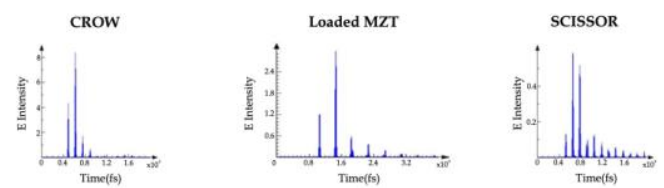

Ring radius $=5 \mu \mathrm{m}$
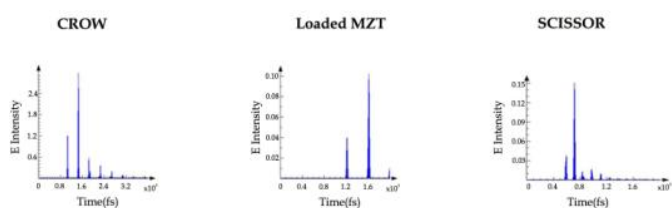

Fig 3: Increment of delay by increasing the ring radius in Time Domain

Output For Rings Of Increasing Radius In Wavelength Domain

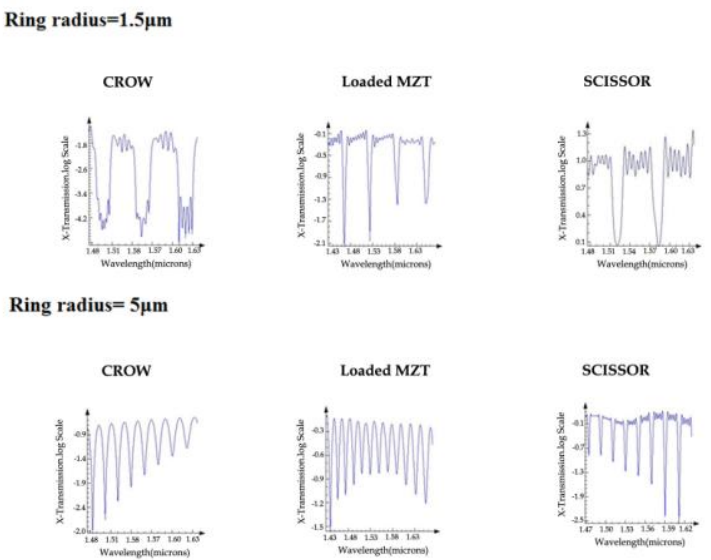

Fig 4: Transmission spectrum for increased ring radius

From table 1 and 2 it is shown that, even though the SCISSOR structure provides delay which is slightly less than that achieved by loaded MZT configuration the later configuration suffers from bending loss at the waveguide bends The CROW structure provides delay which is lower than that of both SCISSOR and Loaded MZT configuration.

Table. 1 and 2 shows that as the ring radius are increased the overall delay and the group delays are also increased. From the filter characteristics it is shown that the increase in the number of rings decreases the Free Spectral Range of the device. 
Output For Waveguides Of Different Width For 10 Rings Of Radius $=5 \mu \mathrm{m}$ In Time Domain

Bus Width = Ring Width
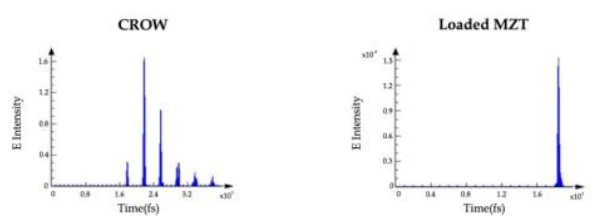

Bus Width > Ring Width crow Loaded MZI
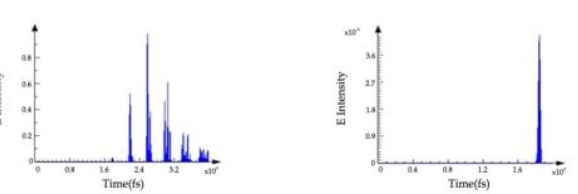

Fig 5: Comparison of time domain outputs for waveguides of same width and different width

Output For Waveguides Of Different Width For 10 Rings Of Radius $=5 \mu \mathrm{m}$ In Wavelength Domain

Bus Width = Ring Width
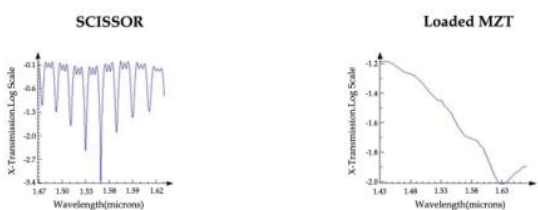

Bus Width > Ring Width
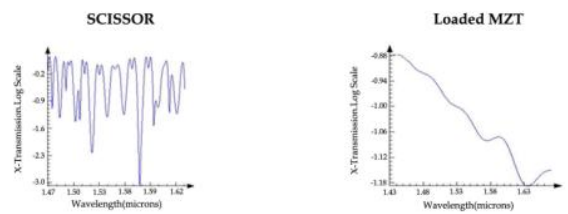

Fig 6: Comparison between the Transmission spectrum of SCISSOR and CROW structures for the waveguides of same width and different width

Table 1. Comparison of Three Delay lines for Ring Radius of $1.5 \mu \mathrm{m}$

\begin{tabular}{|c|c|c|c|}
\hline \multirow{2}{*}{ Parameter } & \multicolumn{3}{|c|}{ Number of Rings - Ring Radius = } \\
\cline { 2 - 4 } & Crow & Scissor & Loaded MZT \\
\hline $\begin{array}{c}\text { Total Delay } \\
\text { (ps) }\end{array}$ & 0.6 & 0.7 & 0.8 \\
\hline $\begin{array}{c}\text { Group Delay } \\
\text { (ps) }\end{array}$ & 0.12 & 0.1 & 0.11 \\
\hline $\begin{array}{c}\text { Loss (dB) } \\
\text { FNR (nm) }\end{array}$ & 1.5 & 0.03 & 0.2 \\
\hline $\begin{array}{c}\text { Bandwidth } \\
(\mathrm{nm})\end{array}$ & 29 & 64 & 65 \\
\hline $\begin{array}{c}\text { Rejection } \\
\text { Line Width } \\
(\mathrm{nm})\end{array}$ & 22 & 24 & 40 \\
\hline \multicolumn{2}{|c|}{} \\
\hline
\end{tabular}

Table 2. Comparison of Three Delay lines for Ring Radius of $5 \mu \mathrm{m}$

\begin{tabular}{|c|c|c|c|}
\hline \multirow{2}{*}{ Parameter } & \multicolumn{3}{|c|}{ Number of Rings - Ring Radius = } \\
\cline { 2 - 4 } & Crow & Scissor & Loaded MZT \\
\hline $\begin{array}{c}\text { Total Delay } \\
\text { (ps) }\end{array}$ & 1.3 & 1.45 & 1.65 \\
\hline $\begin{array}{c}\text { Group Delay } \\
\text { (ps) }\end{array}$ & 0.4 & 0.38 & 0.38 \\
\hline $\begin{array}{c}\text { Loss (dB) } \\
\text { FNR (nm) }\end{array}$ & 0.6 & 0.34 & 0.4 \\
\hline $\begin{array}{c}\text { Bandwidth } \\
\text { (nm) }\end{array}$ & 10 & 1 & 17 \\
\hline $\begin{array}{c}\text { Rejection } \\
\text { Line Width } \\
\text { (nm) }\end{array}$ & 5 & 6 & 10 \\
\hline
\end{tabular}

Table 3. Comparison of Two Delay lines for Equal Bus and Ring Width

\begin{tabular}{|c|c|c|}
\hline \multirow{2}{*}{ Parameter } & \multicolumn{2}{|c|}{$\begin{array}{c}\text { Ring Radius }=\mathbf{5} \boldsymbol{\mu m} \text {, No.of.Rings }=\mathbf{1 0} \\
\text { Bus Width }=\text { Ring Width }\end{array}$} \\
\cline { 2 - 3 } & Scissor & Loaded MZT \\
\hline $\begin{array}{c}\text { Total Delay } \\
\text { (ps) }\end{array}$ & 2.1 & 2.2 \\
\hline $\begin{array}{c}\text { Group Delay } \\
\text { (ps) }\end{array}$ & 0.3 & 0.2 \\
\hline \begin{tabular}{c} 
Loss (dB) \\
\hline $\begin{array}{c}\text { FNR (nm) } \\
\text { Bandwidth } \\
(\mathrm{nm})\end{array}$
\end{tabular} & 0 & 3 \\
\hline $\begin{array}{c}\text { Rejection } \\
\text { Line Width } \\
(\mathrm{nm})\end{array}$ & 6 & 1 \\
\hline
\end{tabular}

Table.3and 4 provides the comparison of the SCISSOR and Loaded MZT delay lines based on the difference in the width of the bus and ring radius. It show that as the width of the ring is greater than that of the bus waveguide the delay in increased in the order of femto seconds.

\subsection{Applications}

These delay lines are used for synchronization. In OTDM demultiplexing low rate control signal is used to demultiplex the high rate data stream. The control and data streams have be aligned within the pulse width and synchronized using delay lines. In antenna Beam Forming Systems, the signals received by each element of the Phased Array Antenna are time-delayed version of some desired satellite signal, and time-delayed version of undesired signal. OBFS delay the entire signals by the appropriate amount of time to synchronize all desired signals and obtain constructive interference. 
Table 4. Comparison of Two Delay lines for Unequal Bus and Ring Width

\begin{tabular}{|c|c|c|}
\hline \multirow[t]{2}{*}{ Parameter } & \multicolumn{2}{|c|}{$\begin{array}{c}\text { Ring Radius }=5 \mu \mathrm{m} \text {, No.of.Rings }=10 \\
\text { Bus Width }<\text { Ring Width }\end{array}$} \\
\hline & Scissor & Loaded MZT \\
\hline $\begin{array}{c}\text { Total Delay } \\
(\mathrm{ps})\end{array}$ & 2 & 2.25 \\
\hline $\begin{array}{c}\text { Group Delay } \\
(\mathrm{ps})\end{array}$ & 0 & 0.4 \\
\hline Loss $(\mathrm{dB})$ & 0.4 & 0.7 \\
\hline FNR (nm) & 1 & 18 \\
\hline $\begin{array}{l}\text { Bandwidth } \\
\quad(\mathrm{nm})\end{array}$ & 5 & 3 \\
\hline $\begin{array}{l}\text { Rejection } \\
\text { Line Width } \\
\text { (nm) }\end{array}$ & 8 & 13 \\
\hline
\end{tabular}

\section{CONCLUSION}

In summary, the paper demonstrates that using cascaded array of micro ring resonators the delay can be increased than that of single resonator. The delay achieved by this design is in the order of Pico seconds for the input pulses of femtosecond width. If the ring waveguide width is larger than that of the bus waveguide the delay can be increased. The gap between the rings also determines the achievable delay. As the ring radius increases the delay also increases linearly. This design can be extended to achieve more delay by cascading more rings and by increasing the ring radius in future.

\section{REFERENCES}

[1] Suphanchai Punthawanunta, Chat Teeka b, Rangson Jomtarak, Preecha P.Yupapind, 2011 Simulation of an Optical Buffer using micro ring Resonator Array with $1.5 \mu \mathrm{m}$ Radius, Science direct, Procedia Engineering 428-431.

[2] L.Y.Mario, and M.K.Chin, 2008 Optical buffer with higher delay bandwidth product in a two-ring system, Opt. Express, vol.16, pp.1796-1807.

[3] V.Van, T. N. Ding, W. N. Herman, and P.T.Ho, J 2008 Group delay enhancement in circular arrays of micro ring resonators," IEEE Photon.Technol. Lett. vol. 20 , no. 12 , pp. 997-999.

[4] F. Xia, L. Sekaric and Y.Vlasov, 2007 Ultra compact optical buffers on a silicon chip, Nat. Photon., vol. 1, pp. 65-71.

[5] Y.Dumeige, 2009 Time-domain analysis of resonator array buffers," IEEE Photon. Techn. Lett. vol. 21, no. 7, pp. 435-437.

[6] G. Lenz, B. J. Eggleton, C. K. Madsen, and R. E. Slusher, 2001 "Optical delay lines based on optical filters," IEEE J.Quantum Electron, vol. 37, no.4, pp. 525-532.

[7] A.D.McAulay, M.R.Corcoran, 2004 "Optical Micro ring resonator filter design trade-offs", SPIE 5595-48. 